\title{
The patent ductus arteriosus management debate: it's not over yet
}

\author{
Susan M. Lopata ${ }^{1} \cdot$ James C. Slaughter ${ }^{2} \cdot$ Maria Gillam-Krakauer $^{1} \cdot$ Jeff Reese $\mathbb{C}^{1,3}$
}

Received: 23 March 2021 / Revised: 25 March 2021 / Accepted: 29 March 2021 / Published online: 15 April 2021

(c) The Author(s), under exclusive licence to Springer Nature America, Inc. 2021

A significant challenge in the care of the premature neonate is reducing the incidence of chronic lung disease (CLD). Efforts to tackle this challenge have included various respiratory support strategies, pharmacological interventions (caffeine, vitamin A, steroids), and active measures to induce closure of a patent ductus arteriosus (PDA) [1]. Prior studies have shown an association between a PDA and the development of CLD [2-6]. Despite these observations, studies evaluating various pharmacologic therapies used for ductal closure given both prophylactically and after evidence of the development of a hemodynamically significant PDA have generally failed to show a decrease in CLD and other adverse outcomes [7]. Neonatologists are left with the impression that strategies to close the ductus have limited benefit and may expose infants to unnecessary treatments and their associated risks. Although non-pharmacologic management strategies have gained popularity [8], there is insufficient information to know whether conservative or non-treatment approaches to a PDA are really the answer. In this issue of the Journal of Perinatology, Bussmann and colleagues report the results of a sub-analysis of The PDA RCT, a single-center double-blind study that found no difference in the primary outcome of CLD or death between infants with high risk for persistent PDA shunt at a gestational age of $<29$ weeks who were treated with ibuprofen $(n=30)$ compared to placebo $(n=30)$ [9]. In the present study, a post hoc secondary analysis found that when infants in the treatment arm were stratified based on

Jeff Reese

jeff.reese@vanderbilt.edu

1 Departments of Pediatrics, Vanderbilt University School of Medicine and the Monroe Carell Jr. Children's Hospital at Vanderbilt, Nashville, TN, USA

2 Biostatistics, Vanderbilt University School of Medicine and the Monroe Carell Jr. Children's Hospital at Vanderbilt, Nashville, TN, USA

3 Cell and Developmental Biology, Vanderbilt University, Nashville, TN, USA successful closure of the PDA, significantly lower rates of the composite CLD/death outcome were observed in infants with a closed ductus (29\%) compared to those with unsuccessful PDA closure $(85 \%)$ or those who received placebo $(60 \%)$. The authors conclude that early shunt elimination may reduce CLD [10]. Further research validating this conclusion should be undertaken given the small sample size of the study ( $n=17$ closed DAs vs. $n=13$ with persistent PDA in the treatment arm) which limits the power of the analysis and its broad applicability. While it may be tempting to include this new information with curated lists of RCTs of PDA treatment [11], the reliance on subanalysis of responders/non-responders in the intervention arm is conceptually problematic, losing the benefits of randomization and blind comparison groups, introducing unseen new confounders, and widening demographic gaps between the new comparison groups that may not be adequately resolved by regression analyses [12]. A strength of this study is the demonstration of the usefulness of a welldefined PDA scoring system in the debate on effect of PDA treatment on respiratory morbidity.

A shared definition of a hemodynamically significant PDA is critical in neonatology for both research and clinical practice in order to both identify infants at greatest risk of morbidity from a prolonged ductal shunt and the efficacy and timing of treatment [13]. Decades-long efforts to develop clinical and echocardiographic criteria that discriminate between the innocent, soon-to-close PDA and the truly hemodynamically significant PDA that poses risk for end-organ injury have led to complex scoring systems [14], but only a few that have been tested in RCTs. To their credit, in this study, El-Khuffash, McNamara, and colleagues nicely demonstrate implementation of a PDA Severity Score (PDAsc) incorporating gestational immaturity and indicators of pulmonary over circulation and left ventricular diastolic function [15]. Clinical decision-making tools such as the PDAsc are essential to guide treatment and improve uniformity in patient recruitment and outcomes measurement in clinical trials. Bussmann and colleagues' PDAsc identified infants with low spontaneous closure rates by 
8 days of age (17\%) in the placebo arm, suggesting that groups with prolonged exposure to a high-volume shunt can be identified. Conversely, there was no difference in the primary CLD/death outcome between the successful ductal closure and low risk no intervention group. Although there is no consensus definition for a hemodynamically significant PDA, the authors' goal to refine the PDAsc will be welcomed by investigators and clinicians alike.

The ideal timing of indicated treatment remains elusive. Infants in The PDA RCT underwent methodical evaluation of the PDA at three separate time points, beginning at 36-48 $\mathrm{h}$ after birth. Infants determined to be at high risk for a hemodynamically significant PDA received treatment at the early time point. Could the association of treatment responders with decreased CLD be associated with early, successful closure of the PDA? Both prophylactic and indicated treatment strategies are currently utilized to manage the ductus. These approaches are met with mixed outcomes data. The TIPP trial found that prophylactic indomethacin may increase CLD in infants without a PDA, which was thought to be related to drug side effects on oxygenation and fluid balance/edema [16]. Assessment of prophylactic indomethacin use in hospitals participating in the National Institute of Child Health and Human Development Neonatal Research Network showed no difference in CLD, although their post hoc analysis suggested possible benefit $[17,18]$. In addition to the current evidence and perceived benefit, the choice to prophylactically treat is undoubtedly influenced by the resources available at a center including access to echocardiograms and interventional cardiology and surgical services [18]. Regarding indicated treatment, the PDA-TOLERATE study compared routine pharmacologic treatment at 1 week for a moderateto-large PDA with conservative therapy. There was no benefit to treatment [19]. A large percentage $(18 \%)$ of patients were treated outside the trial because of lack of physician equipoise. Separate examination of the nonenrolled infants found this subpopulation received earlier treatment and experienced lower rates of respiratory morbidity [20]. This study highlighted the urgency for further research on PDA treatment and timing and the necessity for neonatologists to retain equipoise in order that the appropriate studies can be performed.

Additional research on the ductus and its association with the development of CLD continues to raise even more questions. How could the results of prior research be affected by stratification of treatment recipients into responders versus non-responders? What is the best way to identify infants at greatest risk for significant morbidity related to prolonged ductal shunt? Is waiting until an infant shows symptoms of a hemodynamically significant PDA too late? It remains clear that certain premature infants will benefit from shunt elimination. Continued research is needed to develop an evidence-based approach to determine which infants will benefit from PDA closure and the optimal timing of intervention. Future studies would benefit from incorporation of clinical severity scoring systems, serial echocardiograms, and a priori primary endpoints based on response to treatment.

\section{Compliance with ethical standards}

Conflict of interest The authors declare no competing interests.

Publisher's note Springer Nature remains neutral with regard to jurisdictional claims in published maps and institutional affiliations.

\section{References}

1. Jensen EA. Prevention of bronchopulmonary dysplasia: a summary of evidence-based strategies. Neoreviews. 2019;20:e189-e201.

2. Sellmer A, Bjerre JV, Schmidt MR, McNamara PJ, Hjortdal VE, Høst B, et al. Morbidity and mortality in preterm neonates with patent ductus arteriosus on day 3. Arch Dis Child Fetal Neonatal Ed. 2013;98:F505-510.

3. Palta M, Gabbert D, Weinstein MR, Peters ME. Multivariate assessment of traditional risk factors for chronic lung disease in very low birth weight neonates. The Newborn Lung Project. J Pediatr. 1991;119:285-92.

4. Saldeño YP, Favareto V, Mirpuri J. Prolonged persistent patent ductus arteriosus: potential perdurable anomalies in premature infants. J Perinatol. 2012;32:953-8.

5. Schena F, Francescato G, Cappelleri A, Picciolli I, Mayer A, Mosca F, et al. Association between hemodynamically significant patent ductus arteriosus and bronchopulmonary dysplasia. J Pediatr. 2015;166:1488-92.

6. Mirza H, Garcia J, McKinley G, Hubbard L, Sensing W, Schneider J, et al. Duration of significant patent ductus arteriosus and bronchopulmonary dysplasia in extremely preterm infants. $\mathrm{J}$ Perinatol. 2019;39:1648-55.

7. Sankar MN, Bhombal S, Benitz WE. PDA: to treat or not to treat. Congenit Heart Dis. 2019;14:46-51.

8. Smith A, McNamara PJ, El-Khuffash AF. Non-pharmacological management of a hemodynamically significant patent ductus arteriosus. Semin Fetal Neonatal Med. 2018;23:245-9.

9. El-Khuffash A, Bussmann N, Breatnach CR, Smith A, Tully E, Griffin J, et al. A pilot randomized controlled trial of early targeted patent ductus arteriosus treatment using a risk based severity score (The PDA RCT). J Pediatr. 2021;229:127-33.

10. Bussmann N, Smith A, Breatnach CR, McCallion N, Cleary B, Franklin $\mathrm{O}$, et al. Patent ductus arteriosus shunt elimination results in a reduction in adverse outcomes: a post hoc analysis of the PDA RCT cohort. J Perinatol. 2021. https://doi.org/10.1038/s41372021-01002-Z

11. Sankar MN, Benitz WE. Does crossover treatment of control subjects invalidate results of randomized trials of patent ductus arteriosus treatment? J Perinatol. 2020;40:1863-70.

12. Friedman LM, Furberg C, DeMets DL, Reboussin D, Granger CB Fundamentals of clinical trials/Lawrence M. Friedman, Curt D. Furberg, David L. DeMets, David M. Reboussin, Christopher B. Granger, Fifth edition. edn. Springer: Cham, 2015.

13. Smith A, El-Khuffash AF. Defining "Haemodynamic Significance" of the patent ductus arteriosus: do we have all the answers? Neonatology. 2020;117:225-32. 
14. McNamara PJ, Sehgal A. Towards rational management of the patent ductus arteriosus: the need for disease staging. Arch Dis Child Fetal Neonatal Ed. 2007;92:F424-427.

15. El-Khuffash A, James AT, Corcoran JD, Dicker P, Franklin O, Elsayed YN, et al. A patent ductus arteriosus severity score predicts chronic lung disease or death before discharge. J Pediatr. 2015;167:1354-61.e1352.

16. Schmidt B, Roberts RS, Fanaroff A, Davis P, Kirpalani HM, Nwaesei $C$, et al. Indomethacin prophylaxis, patent ductus arteriosus, and the risk of bronchopulmonary dysplasia: further analyses from the Trial of Indomethacin Prophylaxis in Preterms (TIPP). J Pediatr. 2006;148:730-4.

17. Jensen EA, Dysart KC, Gantz MG, Carper B, Higgins RD, Keszler $M$, et al. Association between use of prophylactic indomethacin and the risk for bronchopulmonary dysplasia in extremely preterm infants. J Pediatr. 2017;186:34-40.e32.

18. Reese J, Shelton EL, Slaughter JC, McNamara PJ. Prophylactic indomethacin revisited. J Pediatr. 2017;186:11-14.e11.

19. Clyman RI, Liebowitz M, Kaempf J, Erdeve O, Bulbul A, Håkansson S, et al. PDA-TOLERATE trial: an exploratory randomized controlled trial of treatment of moderate-to-large patent ductus arteriosus at 1 week of age. $\mathrm{J}$ Pediatr. 2019;205:41-48.e46.

20. Liebowitz M, Katheria A, Sauberan J, Singh J, Nelson K, Hassinger DC, et al. Lack of equipoise in the PDATOLERATE trial: a comparison of eligible infants enrolled in the trial and those treated outside the trial. J Pediatr. 2019;213:222-226.e222. 